\title{
ACANTHOCÉPHALES DE PLEURONECTIFORMES MÉDITERRANÉENS (GOLFE DU LION)
}

\section{Création du genre Solearhynchus (Palæacanthocephala)}

\author{
I. de BURON et C. MAILLARD*
}

RÉSUMÉ. Une étude des Acanthocéphales de Soleidae permet de proposer dans la sous-famille des Echinorhynchinae la création d'un nouveau genre Solearhynchus, avec S. soleae (Porta, 1905) n. comb. parasite de Solea impar, pour espèce type.

\section{Acanthocephalan in Mediterranean flatfishes (Gulf of Lyons). I. Creation of the genus Solearhynchus (Palæacanthocephala).}

SUMMARY. After a study of Acanthocephalan in Soleidae, we propose in the sub-family of the Echinorhynchinae, the creation of a new genus Solearhynchus, with S. soleae (Porta, 1905) n. comb., parasite of Solea impar, as type species.

\section{Introduction}

Les Acanthocéphales de Poissons en Méditerranée n'ont pas fait récemment l'objet d'études taxinomiques suivies. L'inventaire systématique de la parasitofaune des Pleuronectiformes que nous avons entrepris nous a permis de retrouver chez Solea impar Bennett, 1831, Acanthocephaloides soleae (Porta, 1905) Petrotschenko, 1956. Nous proposons de considérer ce parasite comme l'espèce type d'un nouveau genre Solearhynchus.

Chez Solea vulgaris Quensel, 1806, nous avons trouvé quelques individus attribués provisoirement à Acanthocephaloides propinquus (Dujardin, 1845) Meyer, 1932, ainsi qu'une autre espèce qui paraît entièrement nouvelle.

Ce travail a été effectué dans le cadre de l'Équipe de Recherches «Écologie des Plathelminthes parasites 》 de l'Université de Montpellier II et de l'Université de Perpignan, associée au C.N.R.S. (U.A. No 698).

* Laboratoire de Parasitologie comparée (Pr L. Euzet) U.S.T.L., place E.-Bataillon, F 34060 Montpellier Cedex.

Accepté le I3 décembre r983. 
Chez un Bothidae, Arnoglossus laterna (Walbaum, 1792), nous avons récolté un quatrième Acanthocéphale, dont l'espèce, avec les deux précédentes fera l'objet d'une étude ultérieure.

Solearhynchus soleae (Porta, 1905) n. comb.

- Description basée sur 10 femelles dont 1 immature et 7 mâles.

- Hôte : Solea impar Bennett, 1831

- Lieu : Le Grau du Roi (mer Méditerranée)

Types déposés au Muséum National d'Histoire Naturelle sous les numéros : 475 HB HI 105, 475 HB HI 106, 475 HB H35.

Synonyme DE :

- Echinorhynchus soleae Porta, 1905

- E. rhytidodes Monticelli, 1905

- E. aurantiacus Monticelli, 1887 (?)

- E. corrugatus Monticelli, 1900 (?)

- Acanthocephaloides soleae (Porta, 1905) Petrotschenko, 1956

CARACTÈRES GÉNÉRAUX :

- Corps très allongé, cylindrique, toujours inerme.

- Rostre ovoïde, portant 12 files de 6 crochets d'un seul type et à racine vraie.

- Présence de 2 papilles sensorielles sur le cou qui mesure environ $120 \mu \mathrm{m}$ (80-150).

- Ganglion cérébroïde toujours au fond du réceptacle du proboscis. Celui-ci possède une double paroi et mesure $600 \mu \mathrm{m}$ (500-700) de longueur sur $190 \mu \mathrm{m}(150-220)$ de largeur.

- Lemnisques généralement plus courts que le réceptacle $(450 \mu \mathrm{m})$ (300-600).

CARACTÈres MÂles $(P l . I)$ :

- Corps long de 6,3 (5,8-7,5) mm et large de 0,75 (0,70-0,80) mm.

- Rostre de 285 (250-300) $\mu \mathrm{m}$ de longueur sur 160 (150-170) $\mu \mathrm{m}$ de diamètre.

- Système génital occupant plus de la moitié postérieure du tronc : 60 (55-60) \%

- 2 testicules accolés en tandem ou le plus souvent séparés, mesurant 400 (280550) $\mu \mathrm{m}$ de longueur sur 270 (180-400) $\mu \mathrm{m}$ de largeur pour le testicule antérieur, et $410(320-450) \mu \mathrm{m}$ de longueur sur 260 (190-290) $\mu \mathrm{m}$ de largeur pour le testicule postérieur.

- 6 glandes cémentaires le plus souvent régulièrement disposées par paires et très éloignées des testicules. 
— Organe de Säfftigen volumineux : $250 \mu \mathrm{m}$ de diamètre.

- Pénis conique qui débouche dans une cloche copulatrice volumineuse.

- Orifice génital terminal.

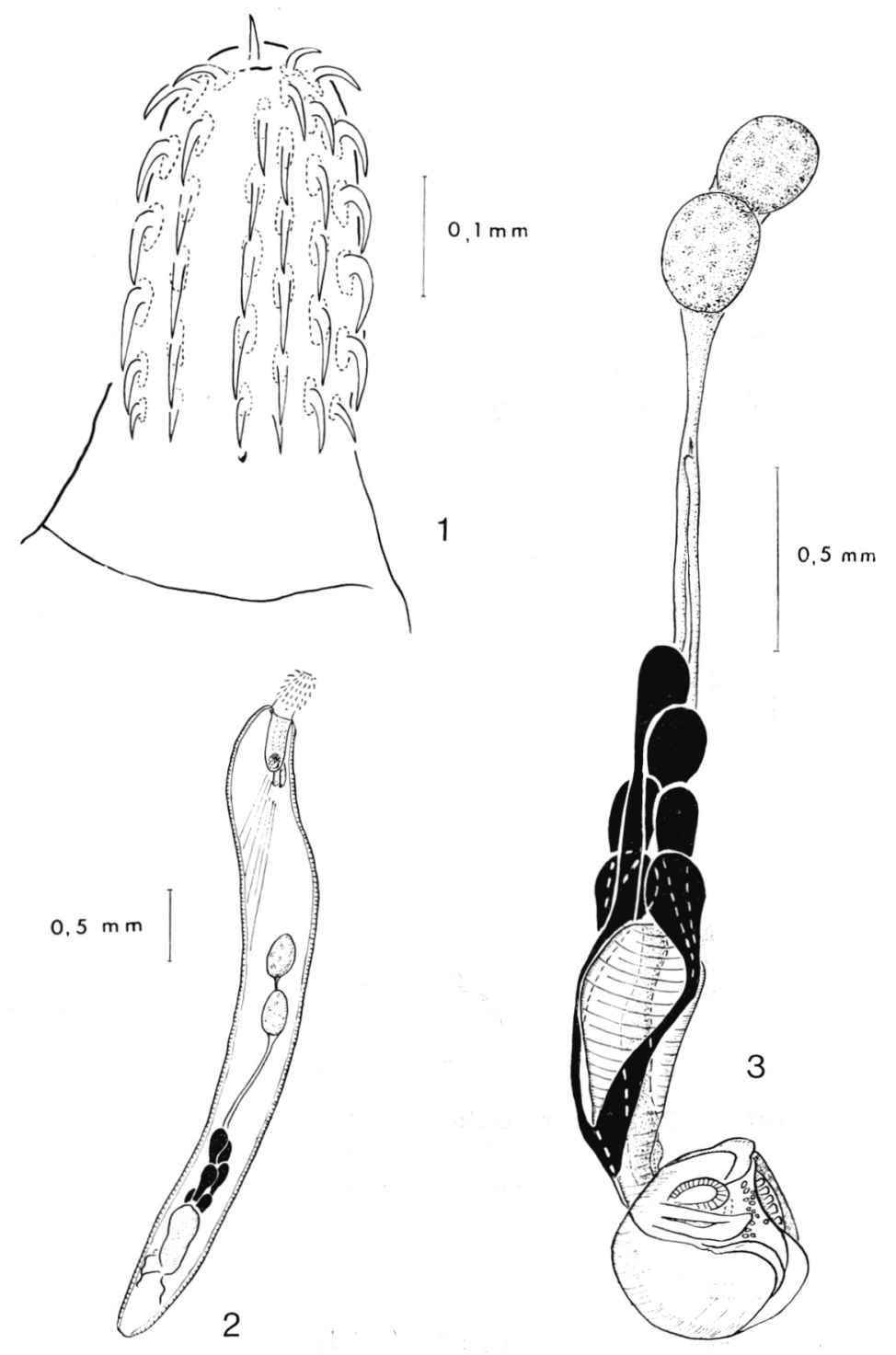

Planche I. - Solearhynchus soleae (Porta, 1905) n. comb.

Individu mâle :

I : Rostre ; 2 : Individu in toto ; 3 : Système génital. Cloche copulatrice évaginée. 

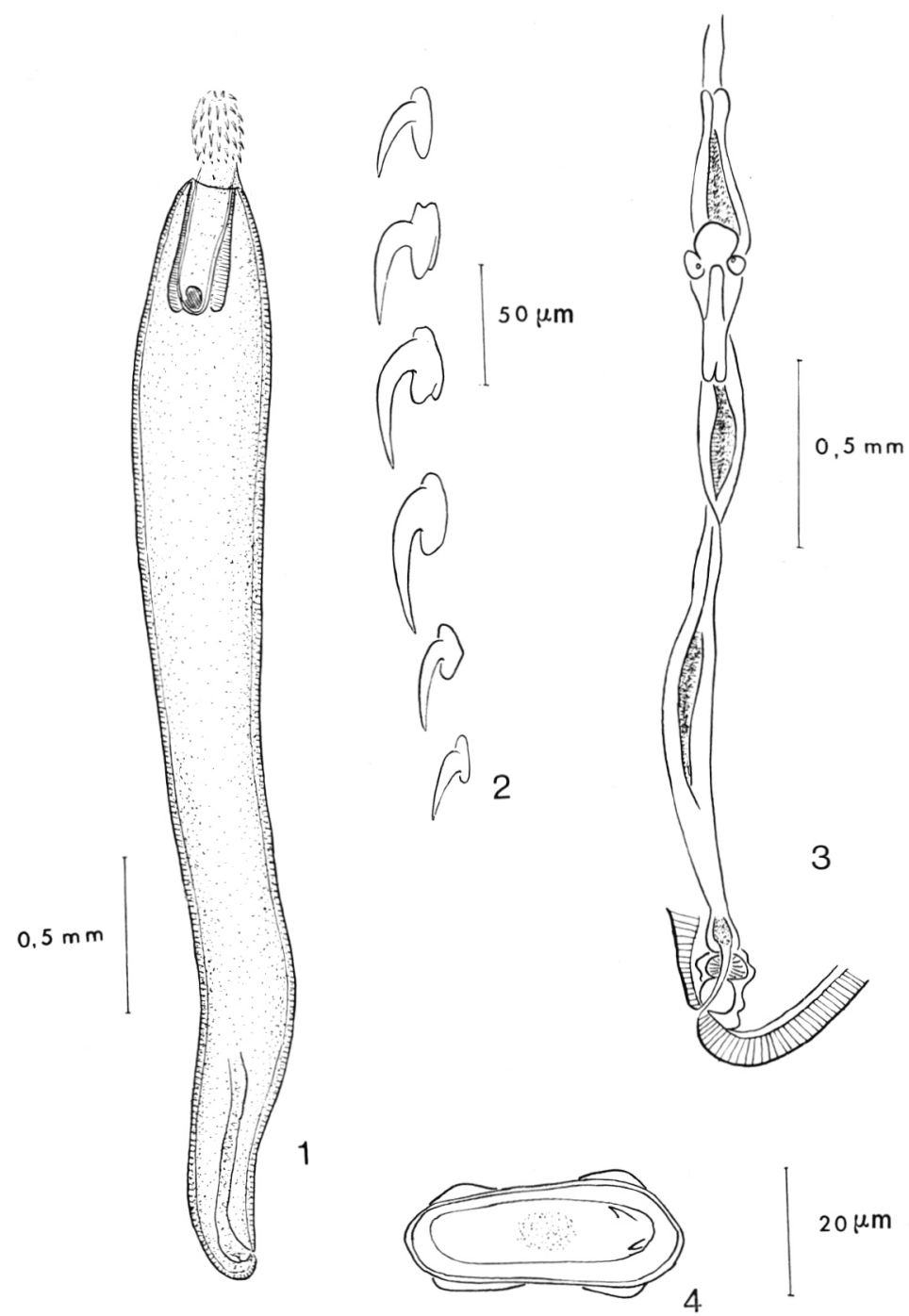

Planche II. - Solearhynchus soleae (Porta, I905) n. comb.

Individu femelle :

I : Individu in toto ; 2 : Crochets du rostre ; 3 : Système génital ; 4 : Embryophore mûr contenant la larve acanthor.

Caractères femelles $(P l . I I)$ :

- Corps long de 7 (5-10) $\mathrm{mm}$ et large de 0,7 (0,5-1) $\mathrm{mm}$.

- Rostre très ovoïde, mesurant $310(280-350) \mu \mathrm{m}$ de longueur sur $180(150-200) \mu \mathrm{m}$ de diamètre.

- Un unique sphincter vaginal. 
- Vulve sub-terminale.

- Embryophore très petit : $40 \mu \mathrm{m}$ de longueur sur $15 \mu \mathrm{m}$ de diamètre et présentant des ornementations très caractéristiques, c'est-à-dire 4 dilatations latérolongitudinales.

\section{Discussion}

Porta en 1905 décrit sous le nom de Echinorhynchus soleae un Acanthocéphale parasite de Solea impar en mer Méditerranée (côtes italiennes). D'après Golvan, 1969, la description et l'iconographie sont très sommaires. Meyer (1931) classe cette espèce dans les Echinorhynchus sensu lato ; Petrotschenko (1956) l'inclut dans le genre Acanthocephaloides malgré le caractère inerme des individus décrits par Porta et l'existence d'un seul type de crochets de leur proboscis. Or les critères essentiels de la diagnose de ce dernier genre sont l'uniformité des champs d'épines cuticulaires sur tout le tronc et la présence de 2 types très distincts de crochets du rostre.

Après comparaison avec les figures de Monticelli (1905) et les individus recueillis ayant un tronc inerme et possédant un seul type de crochets sur leur rostre tel que le décrivait Porta, nous pensons être en présence de l'espèce Echinorhynchus soleae Porta, 1905.

A cause des glandes cémentaires syncytiales multiples, cet Acanthocéphale appartient à l'ordre des Palceacanthocephala Meyer, 1931.

Parasite de Poissons, nous le plaçons dans la super-famille des Echinorhynchidea (Cobbold, 1876) Golvan et Houin, 1963.

Possédant 6 glandes cémentaires, un seul type de crochets du rostre, un cou de petite taille, il appartient à la famille des Echinorhynchidae Cobbold, 1876.

Deux sous-familles composent cette famille :

- La sous-famille des Yamagutisentinae Golvan, 1969 regroupe des espèces possédant un champ antérieur d'épines cuticulaires; nous ne la considérons donc pas pour identifier l'espèce présente.

- La sous-famille des Echinorhynchinae Cobbold, 1876 regroupe des espèces inermes. Nous pensons donc que l'espèce que nous venons de décrire appartient à cette dernière sous-famille. Celle-ci est composée de 6 genres dont 4 sont parasites de Vertébrés d'eau douce. Seuls les genres Echinorhynchus Müller, 1776 et Metacanthocephaloides Yamaguti, 1959 sont marins.

Cependant, dans ces 2 derniers genres, le ganglion cérébroïde est soit médian, soit antérieur, donc jamais postérieur comme c'est le cas pour les individus que nous récoltons chez Solea impar. Nous pensons donc qu'il s'agit d'un genre différent que nous proposons de nommer Solearhynchus.

\section{DiAgNose DU GENRE}

- Echinorhynchinae parasite de Poissons marins (Soleidae).

- Corps cylindrique de taille moyenne. 
- Proboscis ovoïde armé de crochets d'un seul type et possédant tous de vraies racines.

- Cou portant 2 papilles sensorielles.

- Ganglion cérébroïde placé en fond du réceptacle.

— Système génital mâle occupant plus de la moitié postérieure du tronc.

- Un unique sphincter vaginal.

- Orifice génital terminal chez le mâle et sub-terminal chez la femelle.

EsPèce TYPE : Solearhynchus soleae (Porta, 1905) n. comb.

\section{Conclusion}

L’inventaire des Acanthocéphales des Poissons Pleuronectiformes méditerranéens (golfe du Lion) nous montre que la taxinomie de ces parasites est entièrement à revoir. Cette étude est actuellement en cours à partir de récoltes réalisées chez les Bothidae, les Soleidae, etc... Toutefois, le problème s'avère complexe chez les espèces congénériques de Soleidae, d'autant plus que la systématique de ces hôtes est actuellement en évolution (Quignard, Pasteur et Shehata : communication personnelle).

Remerciements : Nous tenons à remercier MM. les Professeurs Euzet et Golvan pour l'aide qu'ils ont bien voulu apporter à la réalisation de ce travail.

\section{BIBLIOGRAPHIE}

Golvan Y. J. : Systématique des Acanthocéphales (Acanthocephala Rudolphi, I8or). L'ordre des Palaacanthocephala Meyer, I93I. La super-famille des Echinorhynchoidea (Cobbold, I876), Golvan et Houin, I963. Mém. Mus. Nat. Hist. Nat., 1969, 57, fascicule unique, 373 p.

Meyer A. : Neue Acanthocephalen aus dem Berliner Museum, Begründung eines neuen Acanthocephalen Systems auf Grund einer Untersuchung der Berliner Sammlung. Zool. Jahrb. System. Geograph., I931, 62, 53-108.

Monticelli F. S. : Su di un Echinorinco della Collezione del Museo Zoologico di Napoli (Echinovhynchus vhytitodes Montic.). Annuar. Mus. Zoolog. Univ. Napoli (Nuova ser.), I905, I, I-I3.

Petrotschenko V. I. : Acanthocephala of domestic and wild animals. I.P.S.T., I956, I, 465 p. Porta A. : Gli Echinorhynchi dei Pesci. Archiv. Zoolog. Napoli, I905, 2, I49-2 I 4.

Quignard J. P., Pasteur N., Shehata S. : Biosystématique du complexe Solea vulgaris du golfe du Lion (Poissons, Téléostéens, Soléidés). Génétique, morphologie et étude méristique. Rev. Inst. Pêches marit., I982 (1984), 46, 273-284. 